\title{
Atomic-Level Chemical Analysis by EELS and XEDS in Aberration-Corrected Scanning Transmission Electron Microscopy
}

\author{
M. Watanabe ${ }^{1}$, E. Okunishi ${ }^{2}$ and T. Aoki ${ }^{1,3}$ \\ ${ }^{1}$ Dept of Materials Science and Engineering, Lehigh University, Bethlehem. PA 18015 \\ 2 JEOL Ltd, Akishima, Tokyo, 196-8558, Japan. \\ ${ }^{3}$ JEOL USA Ltd. Peabody, MA 01960
}

Chemical analysis at atomic-level spatial resolution with single-atom detection sensitivity is one of the ultimate goals in materials characterization. Such atomic-level materials characterization would be feasible by electron energy-loss spectrometry (EELS) and X-ray energy dispersive spectrometry (XEDS) in the latest aberration-corrected scanning transmission electron microscopes (STEMs) because more probe current can be added into the incident probe by aberration-correction. Especially for EELS analysis, sufficient amounts of core-loss signals can be generated within a short acquisition time by higher current probes, and hence atomic-resolution EELS mapping has already been applied [e.g., 1-3]. For XEDS analysis, spatial resolution reaches $\sim 0.4 \mathrm{~nm}$ [4], which implies atomic-level analysis is feasible, in aberrationcorrected STEM. In this paper, the latest attempts to obtain atomic-level elemental distributions by EELS and XEDS approaches in aberration-corrected STEM will be presented.

For atomic-level analysis, the STEM probe must be positioned above individual atomic column sites during acquisition, which requires relatively long-term instrumental and environmental stabilities. The newly developed JEOL ARM-200F aberration-corrected STEM instrument is designed to perform the atomic-level chemical analysis with improved instrumental stabilities. An HAADF-STEM image from an interface in a $\mathrm{LaMnO}_{3} / \mathrm{SrTiO}_{3}$ multilayer thin-film is shown in Fig. 1(a). The bright and slightly fainter spots appearing in this HAADF-STEM image correspond to heavy atom columns of $\mathrm{La}$ or $\mathrm{Sr}$ and to $\mathrm{Ti}-\mathrm{O}$ or $\mathrm{Mn}-\mathrm{O}$ columns in the perovskite structure, respectively. An EELS spectrum-imaging (SI) data was acquired from the same field of view with $186 \times 26$ pixels and 1350 channels for a dwell time of 0.1 s using a Gatan Enfina spectrometer. After applying multivariate statistical analysis (MSA) [5] to enhance weak signals in the dataset, elemental maps were extracted by power-low background subtraction. From the extracted elemental maps, two RGB color-overlay images were constructed as shown in Fig. 1(b, Red: Ti L $L_{2,3}$, Green: Sr $\mathrm{M}_{2,3}$ and Blue: $\mathrm{O} \mathrm{K}$ ) and 1 (c, Red: $\mathrm{Mn} \mathrm{L}_{2,3}$, Green: La $\mathrm{M}_{4,5}$ and Blue: $\mathrm{O}$ K), which represent $\mathrm{SrTiO}_{3}$ and $\mathrm{LaMnO}_{3}$ layers, respectively. Elemental distributions at individual atomic columns can be clearly distinguished. Especially the $\mathrm{Ti}$ distribution is terminated at the $\mathrm{LaMnO}_{3} / \mathrm{SrTiO}_{3}$ interfaces relatively sharply, whereas the $\mathrm{Mn}$ distribution seems diffused toward $\mathrm{SrTiO}_{3}$ layers.

Atomic-level chemical analysis is even more challenging in the XEDS approach since detection of X-ray signals is more limited than that in EELS ( 100 times difference). An XEDS SI dataset was recorded from a [100]-projected GaAs specimen and then MSA was applied to improve weak signals in the dataset. Figure 2 shows a HAADF-STEM image (a) and X-ray maps of Ga $\mathrm{K} \alpha$ and $\mathrm{As} \mathrm{K} \alpha$ lines (b), and EELS maps of $\mathrm{Ga} \mathrm{L}_{2,3}$ and $\mathrm{As}_{2,3}$ edges (c), which were also recorded with the XEDS SI dataset simultaneously. Although the signal levels are still very limited in comparison to EELS analysis, the atomic-level XEDS analysis is now possible in combination of aberration-corrected STEMs with advanced statistical analysis such as MSA. 


\section{References}

[1] M. Bosman, et al., Phys. Rev. Lett., 99 (2007), 086102.

[2] D.A. Muller, et al., Science, 319 (2008), 1073.

[3] M. Varela, et al., Phys. Rev. B, 79 (2009), 085117.

[4] M. Watanabe, et al., Microsc. Microanal. 12 (2006), 515.

[5] M. Watanabe et al., Microsc. Anal., 23 (2009), Issue 7, 5.

[6] The author (MW) wish to acknowledge the financial support of the NSF through grant (DMR-0804528). We also thank Drs. Maria Varela and Ho Nyung Lee at ORNL who provided the $\mathrm{LaMnO}_{3} / \mathrm{SrTiO}_{3}$ multilayer specimen.

\section{(a) HAADF-STEM image}

Fig. 1

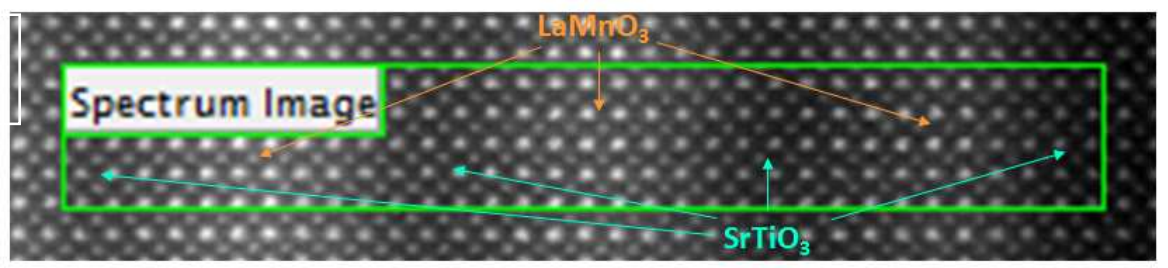

(b) Color-overlaid chemical image of $\mathrm{Ti}$ (red), $\mathrm{Sr}$ (green) and $\mathrm{O}$ (blue)

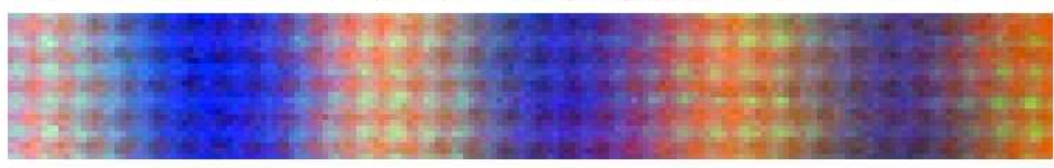

(c) Color-overlaid chemical image of $\mathrm{Mn}$ (red), La (green) and O (blue)

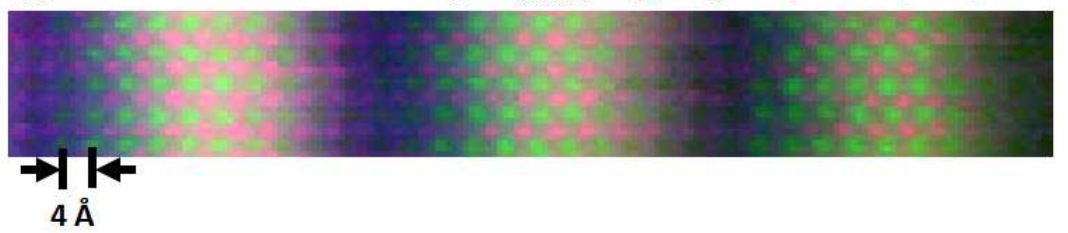

Fig. 2

(a) HAADF-STEM

(b) XEDS maps

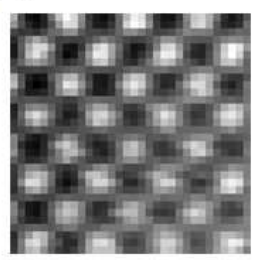

$0.5 \mathrm{~nm}$
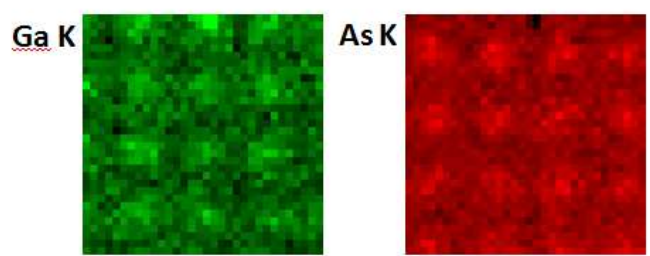

(c) EELS maps
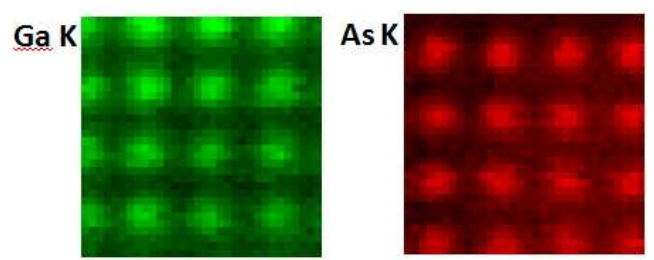

Figure 1: (a) An HAADF-STEM image from a $\mathrm{LaMnO}_{3} / \mathrm{SrTiO}_{3}$ interface, (b) $\mathrm{RGB}$ color-overlay image of $\mathrm{SrTiO}_{3}$ and (c) RGB color-overlay image of $\mathrm{LaMnO}_{3}$, obtained by the EELS approach. Figure 2 (a) An HAADF-STEM image from a GaAs specimen, (b) XEDS elemental maps of Ga $\mathrm{K} \alpha$ and $\mathrm{As} \mathrm{K} \alpha$ lines, and (c) EELS maps of $\mathrm{Ga} \mathrm{L}_{2,3}$ and $\mathrm{As} \mathrm{L}_{2,3}$ edges. 